\title{
Prosthetic limb salvage surgery for bone and soft tissue tumors around the knee
}

\author{
RUI NIIMI, AKIHIKO MATSUMINE, TAKAHIKO HAMAGUCHI, TOMOKI NAKAMURA, \\ ATSUMASA UCHIDA and AKIHIRO SUDO
}

Department of Orthopaedic Surgery, Mie University Graduate School of Medicine, Mie 514-8507, Japan

Received February 20, 2012; Accepted April 9, 2012

DOI: $10.3892 /$ or.2012.2021

\begin{abstract}
In this study, we analyzed long-term survival, limb function and associated complications after prosthetic limb salvage treatment in patients with bone and soft tissue tumors around the knee joint. A total of 63 patients treated with prosthetic limb salvage surgery around the knee were reviewed. The bone tumors involved the distal femur in 45 patients, the proximal tibia in 14 patients and the soft tissue tumors of the proximal lower leg in 4 patients. The median follow-up period after the first operation was 8.0 years. The medical records of the patients, surgical reports, radiographs and histological specimens were retrospectively reviewed. The 5-year overall survival rate was $63.2 \%$ in the patients with distal femur tumors and $86.2 \%$ in those with tumors of the proximal lower leg. The 5-year prosthetic survival rate was $72.8 \%$ in the distal femur and $74.6 \%$ in the proximal lower leg. The mean functional score according to the scoring system of the Musculoskeletal Tumor Society (MSTS) was $81 \%$ in the patients with distal femur tumors and $82 \%$ in the patients with proximal lower leg tumors. Post-operative complications occurred in 27 patients. Limb salvage surgery is considered to be an effective treatment option. However, the high complication rate is a major concern for prosthetic replacement. Future improvements of prostheses are very important.
\end{abstract}

\section{Introduction}

A few decades ago, the primary aim of treatment for sarcoma arising in the extremities was to save the patient's life. Amputation was the standard surgical treatment for musculoskeletal sarcomas of the extremities. In the 1980s, advances in adjuvant therapy improved the tumor control and patient survival, advances in diagnostic imaging allowed more accurate tumor staging and advances in surgical and reconstructive

Correspondence to: Dr Akihiko Matsumine, Department of Orthopaedic Surgery, Mie University Graduate School of Medicine, 2-174 Edobashi, Tsu, Mie 514-8507, Japan

E-mail: matsumin@clin.medic.mie-u.co.jp

Key words: sarcoma, knee joint, reconstruction, function, complications techniques provided limb salvage surgery as a safe alternative to amputation. Today, limb salvage surgery is performed in $70-95 \%$ of all patients with bone and soft tissue sarcoma of the extremities, even if the tumor is high-grade (1-4).

However, reconstruction of a large bone defect after resection of a tumor with a wide margin remains a major challenge. The options for reconstruction after resection of a tumor around the knee joint include implantation of a prosthesis, osteoarticular allograft, allograft-prosthesis composite, recycled autologous bone graft, arthrodesis with intercalary bone grafting or conversion to a rotationplasty (5). Prosthetic replacement after resection of a bone tumor around the knee joint has been demonstrated to provide good function in most cases. However, unfortunately, prosthesis-related complications still remain an unresolved problem. Improvement in patient survival has led to subsequent surgical revisions of the prosthesis as a result of increases in prosthesis-related complications. These include periprosthetic infections, aseptic loosening, and wear of the joint components, dislocations, breakage of the prosthesis and fatigue fractures. The long-term functional outcome of the affected limbs depends on these complications. Until now, these are some reports concerning to the prosthetic limb salvage surgery around the knee.

The purpose of this study was to evaluate the clinical and functional results of patients who underwent a limb salvage surgery for tumors around the knee.

\section{Materials and methods}

Between 1982 and 2008, 63 patients underwent limb salvage surgery. The mean follow-up period was 8.0 years (range, 0.8-28 years) after the initial operation. The patients' medical records, surgical reports, radiographs and pathologic records were retrospectively reviewed (Table I). Written informed consent was obtained from all of the patients included in this study.

The patient group included 34 males and 29 females, with a mean age of 29 years (range, 11-79 years) at the initial treatment. The bone tumors involved the distal femur in 45 patients, the proximal tibia in 14 patients, and the soft tissue tumors of the proximal lower leg in 4 patients. There were 45 high-grade osteosarcomas (OS), 3 chondrosarcomas, 3 malignant fibrous histiocytomas (MFH), 5 giant cell tumors (GCT) and other in 7 patients. According to the Enneking 
staging system (6), one patient was categorized as Stage I, four patients as IIA, 50 patients as IIB, and three patients as III. The surgical excisions were performed with wide margins in all cases. Forty-six patients underwent chemotherapy pre- and post-operatively. The chemotherapy was performed using a combination of 2-4 chemotherapeutic drugs, including cisplatin, doxorubicin, cyclophosphamide, ifosfamide and methotrexate. Post-operative chemotherapy was started 2-3 weeks after surgery. No patient underwent radiotherapy.

Surgical methods. The surgical technique involved resection of the tumor and reconstruction of the knee joint. The biopsy root was also excised en bloc with a margin of $>3-\mathrm{cm}$ in the malignant lesion. The tumor resection was carried out according to standard oncologic principles. In all cases, the surgical excision was performed with a wide margin (5). In the distal femoral tumors, the median resection length was $16 \mathrm{~cm}$ (range, 9-22 cm), and in the proximal tibia tumors, the median resection length was $14 \mathrm{~cm}$ (range, $11-21 \mathrm{~cm}$ ).

Special attention was given to cover the prosthesis completely with muscle tissue. For the distal femur tumors, the remaining vastus medialis was sutured to the rectus femoris. The sartorius muscle could be mobilized and rotated anteriorly for additional closure of the remaining medial soft tissue defect. For the proximal lower leg tumor, reconstruction of the extensor mechanism was performed in all patients. The patellar tendon and anterior capsule were advanced and sutured to the anterior aspect of the prosthesis using unabsorbent thread. A medial gastrocnemius transposition flap (GTF) was used in all cases to provide adequate soft-tissue coverage. In one patient, suturing of the patellar ligament to the anteriorly transferred fibula was performed, and this was then followed by covering the prosthesis with a GTF.

Prosthesis. After resection of the tumor, reconstruction was performed using a prosthesis. Depending on the year the surgery was performed four types of prostheses were used, which were selected depending on the tumor location. From 1982 to 1987 , a custom-made prosthesis manufactured by Nemoto-shokai (Tokyo, Japan) was used. This prosthesis has a restrained hinge and was fixed with cement. Three Nemotoshokai custom-made prostheses were used for reconstruction of distal femurs and six were used for proximal lower legs. From 1988 to 1997, cementless prostheses, HMRS (StrykerHowmedica-Osteonics, Rutherford, NJ), were used for reconstruction of distal femurs. Thirteen HMRS prostheses were used for reconstruction of distal femurs. This type of prosthesis is still being used for the proximal lower leg. In all 12 HMRS prostheses were used for proximal lower legs during the study period. The HMRS is a first-generation modular endoprosthetic system. It features intramedullary, cementless, press-fit stems supported by external flanges and cortical transfixation screws. The knee mechanism consists of a simple hinge design. From 1997, for the reconstruction of the distal femur, 26 patients were treated using the KLS/ PHKIII system (Japan Medical Materials, Osaka, Japan). The $\mathrm{KLS} / \mathrm{PHKIII}$ is an original prosthesis in that the metallic parts are fully made of titanium alloy, and this prosthesis has a unique semi-rotating hinge joint and was designed especially for people with the Asian body type/stature (7). Three patients
Table I. Details of the patient characteristics.

\begin{tabular}{|c|c|c|}
\hline & $\begin{array}{l}\text { Distal femur } \\
\text { (45 patients) }\end{array}$ & $\begin{array}{c}\text { Proximal tibia } \\
\text { (18 patients) }\end{array}$ \\
\hline Age & $30 \quad(11-79)$ & $28(13-68)$ \\
\hline \multicolumn{3}{|l|}{ Gender } \\
\hline Male & 25 & 9 \\
\hline Female & 20 & 9 \\
\hline $\begin{array}{l}\text { Follow-up periods } \\
\text { (months) }\end{array}$ & $78.3(3-340)$ & $141(5-305)$ \\
\hline \multicolumn{3}{|l|}{ Diagnosis $^{\mathrm{a}}$} \\
\hline OS & 35 & 10 \\
\hline $\mathrm{MFH}$ & 1 & 2 \\
\hline Metastasis & 2 & 1 \\
\hline $\mathrm{CS}$ & 3 & 0 \\
\hline GCT & 4 & 1 \\
\hline Others & 0 & 4 \\
\hline \multicolumn{3}{|l|}{ Surgical staging ${ }^{\mathrm{b}}$} \\
\hline 3 & 4 & 1 \\
\hline IA & 0 & 1 \\
\hline IIA & 4 & 0 \\
\hline IIB & 34 & 16 \\
\hline III & 3 & 0 \\
\hline \multicolumn{3}{|l|}{ Prosthesis } \\
\hline Custom made & 3 & 6 \\
\hline Modular & 42 & 12 \\
\hline
\end{tabular}

${ }^{\mathrm{a} O S}$, osteosarcoma; MFH, malignant fibrous histiocytomas; CS, chondrosarcoma; GCT, giant cell tumor. ${ }^{\text {b }}$ tage 3 is gengin aggresive lesion; Stage IA is a low-grade, intracompartmental lesion without metastases; Stage IB is a low-grade, extracompartmental lesion without metastases; Stage IIA is a high-grade, intracompartmental lesion free of metastases; Stage IIB is a high-grade, extracompartmental lesion without metastases; and Stage III is setting with metastases.

with distal femur tumors were implanted with other types of modular prostheses in the 1980s.

Assessment and statistical analysis. The oncological results, functional results, and complications were investigated. A functional evaluation was performed using the scoring system of the Musculoskeletal Tumor Society (MSTS) which consists of six parameters (pain, function, use of walking aids, walking activity, gait and emotional acceptance) (8). The actuarial data for the overall survival rate, disease-free survival rate, prosthesis survival rate and limb salvage rate at the final follow-up were calculated using a Kaplan-Meier analysis. The prosthesis survival rate was calculated as the time from the surgical reconstruction using the prosthesis to revision or amputation due to prosthetic failure. Prosthetic failure was defined as replacement of any parts of the prosthetic components, including minor parts of the prosthesis due to local tumor recurrence, polyethylene bushing failures, breakage of the prosthesis and aseptic loosening or infection. Log-rank analyses were used to determine the factors which influenced the prosthesis survival rate. The factors included tumor locations, patient ages $(<30$ 


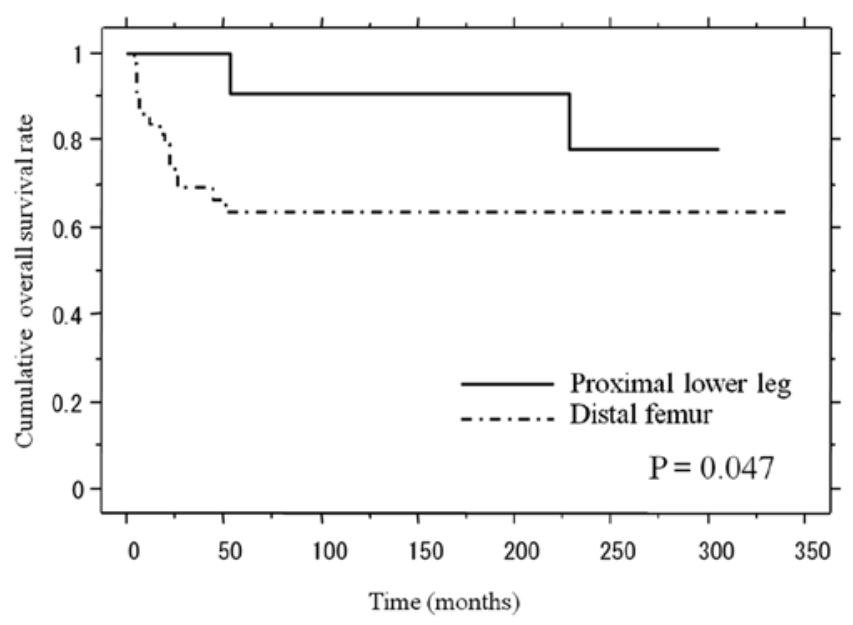

Figure 1. Cumulative overall survival of patients with sarcoma around the knee.

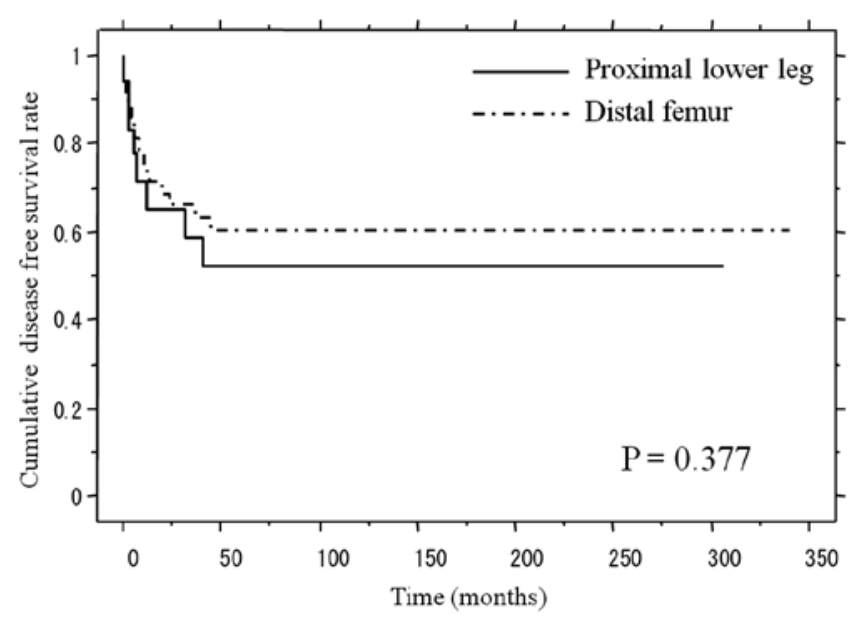

Figure 2. Cumulative disease-free survival of patients with sarcoma around the knee.

vs. $\geq 30$ years), gender, peroneal palsy vs. non-peroneal palsy, extension lag $\left(<30^{\circ}\right.$ vs. $\left.\geq 30^{\circ}\right)$ and primary vs. revision procedures. Mann-Whitney U tests were also used to determine the factors which correlated with the post-operative results of limb function. The factors included tumor locations, patient ages ( $<30$ vs. $\geq 30$ years), gender, perorneal palsy vs. non-peroneal palsy, extension lag $\left(<30^{\circ}\right.$ vs. $\left.\geq 30^{\circ}\right)$ and primary vs. revision procedures. Analyses were performed using the Statview 5.0 software program (SAS Institute, Inc., Cary, NC, USA). A $\mathrm{P}$-value $<0.05$ was considered to be statistically significant.

\section{Results}

Oncological results. At the final follow-up, 36 patients were continuously disease-free, 8 patients had no evidence of disease, two patients alive with disease and 17 were patients dead of disease. The 5-year overall survival rate was $63.2 \%$ in the distal femur cases and $86.2 \%$ in the proximal lower leg cases. The log-rank test showed that there was a significant difference between the cumulative survival rates based on the tumor location ( $\mathrm{P}=0.047$ ) (Fig. 1). The patients with tumors in their

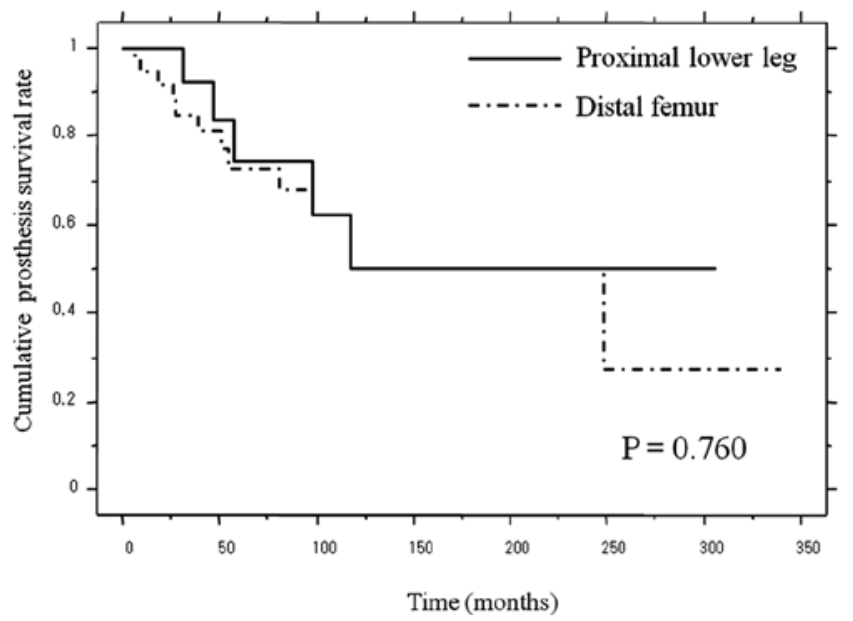

Figure 3. Cumulative prosthetic survival of patients with sarcoma around the knee.

Table II. Results of the univariate analysis showing the factors which affect the 5-year prosthesis survival rate.

\begin{tabular}{lccc}
\hline Factors & $\begin{array}{c}\text { No. of } \\
\text { patients }\end{array}$ & $\begin{array}{c}\text { 5-year prosthesis } \\
\text { survival rate (\%) }\end{array}$ & P-value $^{\mathrm{a}}$ \\
\hline Tumor location & & & \\
$\quad$ Distal femur & 45 & 72.8 & 0.76 \\
$\quad$ Proximal tibia & 18 & 74.6 & \\
Age (years) & & & \\
$\quad<30$ & 31 & 75.3 & 0.91 \\
$\geq 30$ & 32 & 70.3 & \\
Gender & & & \\
$\quad$ Male & 34 & 72.1 & 0.88 \\
Female & 29 & 76.0 & \\
Peroneal nerve palsy & & & \\
$\quad$ Yes & 13 & 83.3 & 0.91 \\
$\quad$ No & 50 & 72.5 & \\
Extension lag & & & \\
$\quad<30^{\circ}$ & 13 & 73.3 & \\
$\geq 30^{\circ}$ & 4 & 0 & \\
\hline
\end{tabular}

${ }^{a}$ Log-rank test.

proximal lower legs had a better overall survival rate than those with distal femoral tumors. The 5-year disease-free survival rates were 62.4 and $52.2 \%$ in the patients with distal femur and the proximal leg tumors, respectively (Fig. 2). There were no significant differences between the disease-free survival rates with regard to the tumor location (log-rank test, $\mathrm{P}=0.377$ ).

Prosthetic survival. The 5-year prosthetic survival rate was $72.8 \%$ in the distal femur cases and $74.6 \%$ in the proximal lower leg cases (Fig. 3). There were no significant differences between the prosthesis survival rates based on the tumor location (log-rank test, $\mathrm{P}=0.760$ ). During the follow-up period, no limb amputation was performed. The log-rank test showed no statistical difference between the prosthetic survival based on 
Table III. Results of the univariate analysis showing the factors which affect the MSTS score.

\begin{tabular}{lccc}
\hline Factors & $\begin{array}{c}\text { No. of } \\
\text { patients }\end{array}$ & $\begin{array}{c}\text { MSTS } \\
\text { score }\end{array}$ & P-value $^{\mathrm{a}}$ \\
\hline Tumor location & & & \\
$\quad$ Distal femur & 45 & $81(40-100)$ & 0.73 \\
$\quad$ Proximal lower leg & 18 & $82(53-97)$ & \\
Age (years) & & & \\
$\quad<30$ & 31 & $81(37-100)$ & 0.85 \\
$\geq 30$ & 32 & $82(40-100)$ & \\
Gender & & & \\
$\quad$ Male & 34 & $86(53-100)$ & 0.04 \\
$\quad$ Female & 29 & $75(37-100)$ & \\
Peroneal nerve palsy & & & \\
$\quad$ Yes & 13 & $66(37-97)$ & 0.01 \\
$\quad$ No & 50 & $85(60-100)$ & \\
Extension lag & & & \\
$\quad<30^{\circ}$ & 13 & $91(73-100)$ & $<0.01$ \\
$\quad \geq 30^{\circ}$ & 4 & $63(37-73)$ & \\
Primary & 46 & $82(37-100)$ & 0.89 \\
Revision & 17 & $78(53-97)$ & \\
\hline
\end{tabular}

${ }^{\text {a Log-rank test. }}$

the following factors: patient's age, tumor location, gender, presence of peroneal nerve palsy and the presence of extension lag (Table II).

Functional results. The mean post-operative functional score was $81 \%$ (range, $37-100 \%$ ). The mean functional score was $81 \%$ (range, $37-100 \%$ ) in the patients with distal femur tumors and $82 \%$ (range, 53-97\%) in the patients with proximal lower leg tumors (Table III).

Regarding the functional results, the Mann-Whitney $U$ test showed that male patients had significantly better limb function than female patients $(\mathrm{P}=0.04)$. Patients with peroneal nerve palsy showed significantly worse MSTS scores than those without peroneal palsy (Mann-Whitney $\mathrm{U}$ test, $\mathrm{P}=0.01$ ). Patients with extension lag in excess of $30^{\circ}$ had a worse MSTS score than those with less extension lag $(\mathrm{P}<0.01)$. The MannWhitney $U$ test showed no significant difference in MSTS scores between primary and revision surgery $(\mathrm{P}=0.89)$.

Complications. A total of 27 of the 63 patients $(43 \%)$ developed post-operative complications. Forty-nine postoperative complications occurred in these 27 patients. The major complications were peroneal palsy in 13 patients $(21 \%)$, followed by loosening in 7 patients $(11.1 \%)$, breakage of the hinge mechanism in 4 patients $(6.3 \%)$, breakage of the stem in 4 patients $(6.3 \%)$, breakage of a femoral or tibial component in 4 patients $(6.3 \%)$, a leg length discrepancy of $>2 \mathrm{~cm}$ in 4 patients $(6.3 \%)$, local recurrence in 4 patients $(6.3 \%)$, deep infection in 3 patients $(4.8 \%)$, skin trouble in 3 patients $(4.8 \%)$, a periprosthetic fracture in 2 patients $(3.2 \%)$, and secondary cancer after chemotherapy in 1 patient (1.6\%) (Table IV).
Table IV. Complications.

Distal femur Proximal tibia

(45 patients) (18 patients)

Prosthesis-related

complications

Breakage of prosthesis

Loosening

Peroneal nerve palsy

Leg length discrepancy

( $\geq 2 \mathrm{~cm}$ )

Recurrence

Deep infection

Skin trouble (only superficial)

Secondary cancer

$\begin{array}{rr}11 & 3 \\ 6 & 1 \\ 5 & 8 \\ 4 & 0 \\ & \\ 3 & 1 \\ 1 & 2 \\ 2 & 1 \\ 1 & 0\end{array}$

Forty-three operations were eventually required to treat these complications. For the deep infections, 19 operations were required, including debridement, primary sutures or coverage with a skin flap. For the skin troubles, 7 minor operations such as secondary suturing were performed. For loosening of the prosthesis, three revision surgeries were required. For the breakage of stems, 4 revision surgeries were performed. Four and 6 surgeries were performed to repair a breakage of a femoral or tibial component, or the breakage of a hinge (e.g., breakage of bush or insert), respectively. Two of the 4 patients with local recurrence underwent a wide excision of the recurrent tumor preserving the prosthesis, and one patient exchanged the prosthesis. Another patient with multiple metastases did not undergo surgery because of a putative poor prognosis.

In cases of distal femoral reconstruction, 6 prostheses, including 3 modular prostheses and 3 custom-made prostheses, were implanted before 1987. Breakage of prosthetic components was observed in 3 of these 6 prostheses $(50 \%)$. Three patients had 4 breakages of their prosthesis. One patient experienced the breakage of a stem. One patient had loosening of a component. One patient had breakage of the hinge after breakage of the stem. But since the HMRS or KLS/PHK III were introduced for reconstruction, the rate of prosthesis breakage was $23 \%$ (9 of 39 prostheses). Nine patients had 13 breakages of the prosthesis.

In addition, in the 6 cases of proximal tibial reconstruction using custom-made prostheses which were implanted before 1987, breakage of the prosthesis was observed in 2 cases (33\%). One patient had breakage of a tibial component, and the other patient had loosening of the prosthesis. But since the HMRS was introduced for reconstruction, the rate of prosthesis breakage has been $17 \%$ ( 2 of 12 patients). One patient suffered the breakage of both the bush and tibial components two times, while another patient had breakage of a tibial component.

\section{Discussion}

In this study, the 5-year overall survival rate was $63 \%$ in the patients with distal femur tumors and $86 \%$ in those with tumors of the proximal lower leg. The 5-year disease-free 


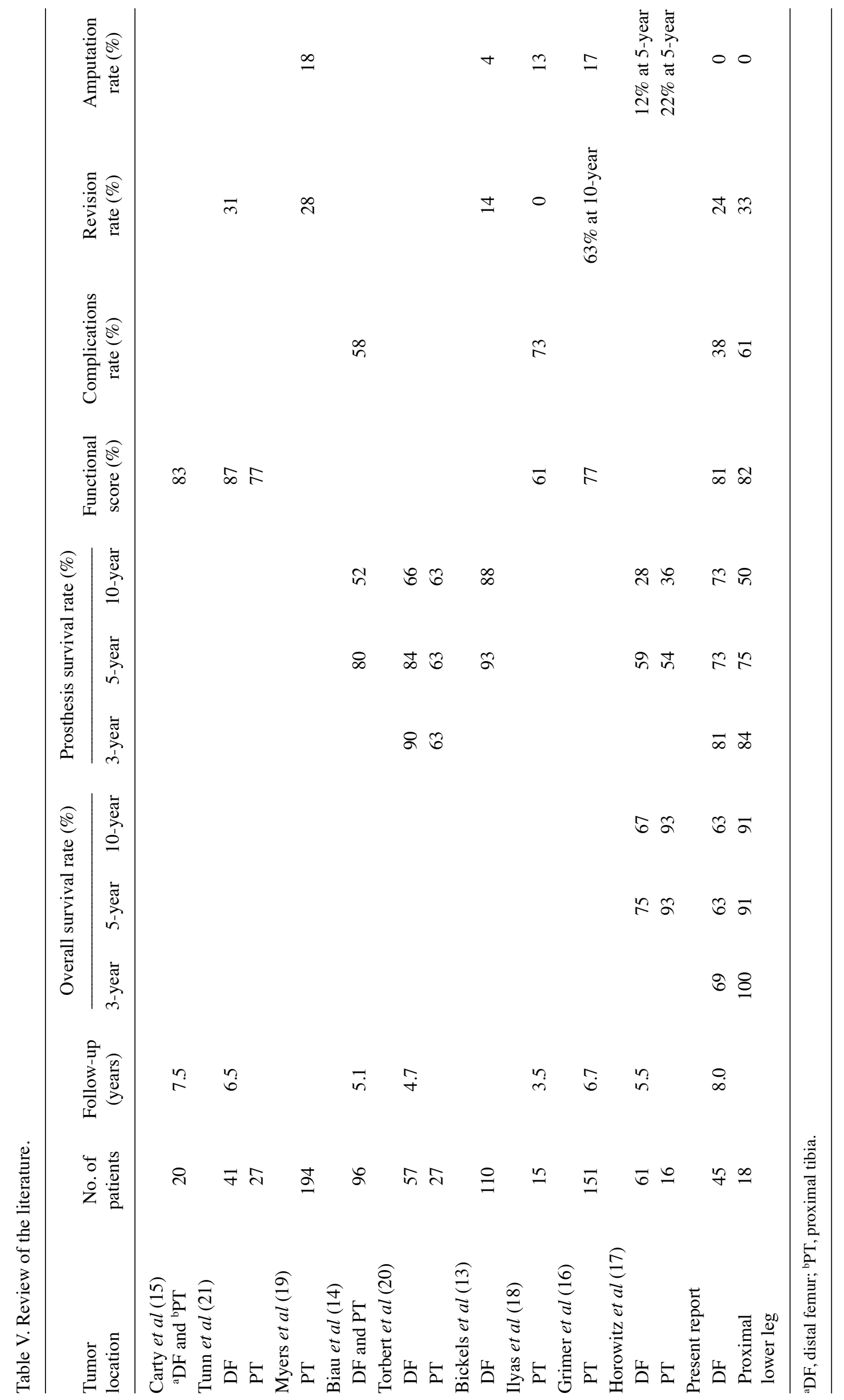


survival rate was 62 and $52 \%$ in the subjects with tumors of the distal femur and the proximal leg, respectively. The 5 -year prosthetic survival rate was $73 \%$ in the distal femur and $75 \%$ in the proximal lower leg. The mean functional score according to the scoring system of the MSTS score was $81 \%$ in the patients with distal femur tumors and $82 \%$ in the patients with proximal lower leg tumors. Post-operative complications occurred in 27 patients (43\%), but no limb amputation was performed.

Both the distal femur and proximal tibia are common anatomic sites for primary and metastatic bone tumors. These tumors were traditionally treated with arthrodesis or amputation of the affected extremity and resulted in unfavorable functional and psychologic outcomes $(9,10)$. However, the prognosis of the sarcoma patients has recently improved as a result of a better understanding of tumor biology, refined chemotherapeutic protocols, advances in diagnostic imaging and improvements in surgical techniques. Improvements in the survival of sarcoma patients made these drawbacks not only more pronounced, but also promoted the development of new surgical reconstructive procedures which promised more useful limb function. Simon et al compared the results of limb-sparing resections to those of amputation in 227 patients with distal femoral osteosarcoma, and concluded that the limb salvage surgery did not shorten the disease-free interval or compromise the long-term survival of these patients compared to amputation (11).

Although amputation still remains one of the important options for patients whose tumors are too advanced to obtain a safe margin, amputation has a negative impact on the patient's psychological well-being. With regard to arthrodesis, it is not suitable for many Asian patients, because squatting is necessary in many components of the Asian lifestyle, such as eating meals while sitting on the floor. Rotationplasty provides good local disease control and good function, but the cosmetic outcome is sometimes not acceptable for patients, especially younger patients. Harris et al also evaluated the results after amputation, arthrodesis and endoprosthetic replacement. They found that only patients with prosthetic reconstruction felt themselves to be close to the healthy population (12). We therefore have pursued limb salvage surgery using a prosthesis as the first choice for resectable malignant tumors occurring around the knee.

In the distal femur, the 3-,5- and 10-year prosthesis survival rates were 85,73 and $68 \%$, respectively. In the proximal lower leg, the 3-, 5- and 10-year prosthesis survival rates were 92, 75 and $50 \%$, respectively. In previous studies, the prosthesis survival of the proximal tibia has been reported to be lower than that of the distal femur (Table V) (13-21). Poor soft tissue coverage, difficulties with anchoring the patellar tendon and possible injuries to the neurovascular system are the most likely causes for this difference. Horowitz et al reported that distal femur prostheses showed 75 and $67 \%$ survival, while proximal tibia prostheses showed 54 and $36 \%$ survival at 5 and 10 years, thus indicating that there were significant differences in the prosthetic survival between distal femur and proximal tibia prostheses (17). Biau et al reported prosthesis 5- and 10-year survival rates of 85 and $55 \%$ in the distal femur, respectively, while they were 72 and $45 \%$ in the proximal tibia (14). In contrast, Torbert et al showed no statistically significant differ- ences in the event-free prosthetic survival rate between the distal femur and proximal tibia prosthesis (20). Distal femur prostheses showed 90, 84 and 66\% survival rate at 3-, 5- and 10 -year, respectively, while proximal tibia prostheses showed $63 \%$ survival rates at 3-, 5- and 10-year. Direct comparisons of the present results to other published reports may be difficult due to the heterogeneity with respect to the patient population and implant used. In our series, log-rank testing showed no significant differences between the prosthetic survival and tumor location. Although the present study consisted of a relatively small number of patients with proximal lower leg tumors, there was no aseptic loosening except for one patient who underwent prosthetic reconstruction before 1987. The HMRS has a porous-coated stem, and permits bone ingrowth at the bone/prosthesis junction. Thus, this prosthetic design might reduce the risk of mechanical failure and loosening, as well as the improvement of the surgical technique.

The rate of infection was $4.8 \%$ in our series, and ranged from $2.2-33 \%$ in previous studies $(13,16,19)$. Infections required secondary procedures. But the consequences nonetheless were noteworthy, with no limb amputation. Infections are difficult to avoid in prosthetic limb salvage surgery for numerous reasons: multiple surgeries, resection of large amounts of tissue, skin sloughing, adjuvant treatments and poor blood supply to the allograft. Providing adequate soft tissue coverage after reconstruction is one of the most critical factors for reducing infection. Grimer et al (16) reported that their initial results for proximal tibia tumor reconstruction were poor because of the high infection rate derived from wound breakdown and an infection rate of $33 \%$. However, this rate later decreased to $12 \%$ with the use of a flap to cover the wound. We therefore recommend the use of a musculocutaneal flap for this operation, though infection, remains a common complication in our study.

In our series, the Mann-Whitney U test showed that male patients had significantly better limb function than female patients. We speculate that this result could be due to the residual muscle power in male patients. Patients with peroneal nerve palsy also showed a significantly worse MSTS score than those without peroneal palsy. These results suggest that preservation of the quadriceps femoris muscle and avoidance of peroneal nerve palsy are critical to maintaining a good limb function.

In summary, prosthetic reconstruction for musculoskeletal tumors around the knee provides good oncological and functional results. However, the high complication rate is a major concern for the prosthetic replacement. Future improvements of prostheses are very important. With regard to the surgical techniques, the preservation of the quadriceps femoralis muscle and avoidance of peroneal nerve palsy are important.

\section{References}

1. Böhm P, Springfeld R and Springer H: Re-implantation of autoclaved bone segments in musculoskeletal tumor surgery. Clinical experience in 9 patients followed for 1.1-8.4 years and review of the literature. Arch Orthop Trauma Surg 118: 57-65, 1998.

2. Niimi R, Matsumine A, Kusuzaki K, Kuratsu S, Araki N, Aoki Y, Ueda T, Kudawara I, Myoui A, Ieguchi M, et al: Usefulness of limb salvage surgery for bone and soft tissue sarcomas of the distal lower leg. J Cancer Res Clin Oncol 134: 1087-1095, 2008.

3. Veth R, Hoesel R, Pruszczynski M, Hoogenhout J, Schreuder B and Wobbes T: Limb salvage in musculoskeletal oncology. Lancet Oncol 4: 343-350, 2003. 
4. Zaretski A, Amir A, Meller I, Leshem D, Kollender Y, Barnea Y, Bickels J, Shpitzer T, Ad-El D and Gur E: Free fibula long bone reconstruction in orthopedic oncology: a surgical algorithm for reconstructive options. Plast Reconstr Surg 113: 1989-2000, 2004.

5. Anract P, Missenard G, Jeanrot C, Dubois V and Tomeno B: Knee reconstruction with prosthesis and muscle flap after total arthrectomy. Clin Orthop Relat Res 384: 208-216, 2001.

6. Enneking WF: A system of staging musculoskeletal neoplasms. Clin Orthop Relat Res 204: 9-24, 1986.

7. Matsumine A, Ueda T, Sugita T, Yazawa Y, Isu K, Kawai A Abe S, Yakushiji T, Hiraga H, Sudo A and Uchida A: Clinical outcomes of the KYOCERA Physio Hinge Total Knee System Type III after the resection of a bone and soft tissue tumor of the distal part of the femur. J Surg Oncol 103: 257-263, 2011.

8. Enneking WF, Dunham W, Gebhardt MC, Malawar M and Pritchard DJ: A system for the functional evaluation of reconstructive procedures after surgical treatment of tumors of the musculoskeletal system. Clin Orthop Relat Res 286: 241-246, 1993.

9. Enneking WF and Shirley PD: Resection arthrodesis for malignant and potentially malignant lesions about the knee using an intramedullary rod and local bone graft. J Bone Joint Surg Am 59: 223-235, 1977.

10. Sugarbaker PH, Malawer MM and Baker AR: Above-knee amputation. In: Musculoskeletal Surgery for Cancer. Sugarbaker PH and Malawer MM (eds). Thieme Medical Publishers, New York, pp229-242, 1992.

11. Simon MA, Aschliman MA, Thomas $\mathrm{N}$ and Mankin HJ: Limbsalvage treatment versus amputation for osteosarcoma of the distal end of the femur. J Bone Joint Surg Am 68: 1331-1337, 1986.

12. Harris IE, Leff AR, Gitelis S and Simon M: Function after amputation, arthrodesis, or arthroplasty for tumors about the knee. J Bone Joint Surg Am 72: 1477-1483, 1990.
13. Bickels J, Wittig JC, Kollender Y, Henshaw RM, KellarGraney KL, Meller I and Malawer MM: Distal femur resection with endoprosthetic reconstruction: a long-term followup study. Clin Orthop Relat Res 400: 225-235, 2002.

14. Biau D, Faure F, Katsahian S Jeanrot C, Tomeno B and Anract P: Survival of total knee replacement with a megaprosthesis after bone tumor resection. J Bone Joint Surg Am 88: 1285-1293, 2006.

15. Carty CP, Dickinson IC, Watts MC Crawford RW and Steadman P: Impairment and disability following limb salvage procedures for bone sarcoma. Knee 16: 405-408, 2009.

16. Grimer RJ, Carter SR, Tillman RM Sneath RS, Walker PS, Unwin PS and Shewell PC: Endoprosthetic replacement of the proximal tibia. J Bone Joint Surg Br 81: 488-494, 1999.

17. Horowitz SM, Glasser DB, Lane JM and Healey JH: Prosthetic and extremity survivorship after limb salvage for sarcoma. How long do the reconstructions last? Clin Orthop Relat Res 293: 280-286, 1993 .

18. Ilyas I, Younge D, Pant R and Moreau P: Limb salvage for proximal tibial tumours using a modular prosthesis. Int Orthop 24: 208-211, 2000

19. Myers GJ, Abudu AT, Carter SR Tillman RM and Grimer RJ: The long-term results of endoprosthetic replacement of the proximal tibia for bone tumours. J Bone Joint Surg Br 89: 1632-1637, 2007.

20. Torbert JT, Fox EJ, Hosalkar HS, Ogilvie CM Ogilvie CM and Lackman RD: Endoprosthetic reconstructions: results of long-term followup of 139 patients. Clin Orthop Relat Res 438: 51-59, 2005.

21. Tunn PU, Pomraenke D, Goerling U and Hohenberger P: Functional outcome after endoprosthetic limb-salvage therapy of primary bone tumours - a comparative analysis using the MSTS score, the TESS and the RNL index. Int Orthop 32: 619-625, 2008. 